\title{
The analysis and application of distance vector routing protocol
}

\author{
Jing Chen \\ Institute of computer engineering, Qiongzhou University, Sanya 572022, China \\ Chenjingmyname@163.com
}

Keywords: routing, Autonomous systems, Gateway protocol, Distance vector.

\begin{abstract}
The routing table is according to the routing protocol for the routing information in routing tables, and is the core of the routing protocol routing algorithms. In practical applications, the routing is a very complicated problem, because it is the result of all the nodes in the network coordination work together. Second, the routing of the environment is always changing, and this kind of change sometimes cannot know in advance, for example, appeared some faults in the network. In addition, when the network congestion occurs, in particular need to ease the congestion of routing strategy, but in these conditions, it is hard to get the required from all nodes in the network routing information. On this basis, put forward based on distance vector routing protocol (RIP), and its analysis and application.
\end{abstract}

\section{Introduction}

Routers forward data packet according to the routing table, so the routing information of the routing table is how to get? And The scale of the Internet is so big that making all of the router know how to get to all the network and routing table will become large, processing is a waste of time; on the other hand, some units is not willing to let the outside world understand its unit network layout details and routing strategy, but also hope to connect to the Internet. So the entire Internet is divided into many smaller units, called Autonomous systems (AS).An AS is also an Internet network, generally belong to the jurisdiction of the administrative unit, the jurisdiction of the department can decide within the AS adopt what kind of routing protocol, and according to the division of the AS Internet, routing protocols can be divided into Internal Gateway Protocol (IGP) and External Gateway Protocol (EGP) two categories.

Routing protocol is the core of the routing algorithms, different routing algorithms usually use different evaluation factors, weight and the calculation of the algorithm for optimal path. Below is the commonly used dynamic routing protocol, introduces routing information protocol RIP configuration and application.

\section{The working principle}

RIPv1 is the first widely used in the internal gateway protocol, it is a distributed vector routing protocol based on distance and its biggest advantage is simple. It requires that every router in the network to maintain distance record from itself to other purpose network distance record, namely distance vector.RIPv1 protocol "distance" is defined that a router to direct connection with network distance as 1 ; a router to a indirectly connected network distance for the number of the transiting router +1 ."+ 1 " because the destination network after direct delivery, and the distance to the direct connection of network is defined as 1 .

RIPv1 protocol of "distance" is known as "Hop Count", because every router, plus one Hop. RIPv1 think that routing is a "good" through the fewer the number of the router, namely "short".RIPv1allows one path only including15routers. Therefore, the maximum "distance" of 16 is inaccessible. It is only applicable to small Internet. RIPv1 protocol can't load balancing feature, at the same time for multiple routing between two networks. RIPv1 select a route having least the router's routing, even if there is another high speed but more routers routing. 


\section{RIPv2}

Distance vector routing protocol uses hop count as a route choice of basic evaluation factor, RIPv1 protocol of convergence process faster, all the nodes in the autonomous system get the correct routing information process. Algorithm implementation simple configuration limitations are relatively simple: Simply use the "hop "evaluation of network path or a lack of comprehensive; Routing updates with neighboring routers on a regular basis for routing information exchange mechanism, easy to form a routing loop; Specified as 15 maximum hop count, have limited the size of the network; There are categories of routing protocol, does not support VLSM network - is designated to participate in the RIP routing updates having the same subnet mask.RIPv2 improves it.

RIPv2 protocol itself does not change much, but the performance is improved, still uses hop count as routing evaluation factor, maximum hop count is still 15. Improvement reflected in the following points: to provide the prefix routing, can send the subnet mask in routing updates information to support in the same autonomous system using VLSM. Provides authentication mechanism in the update information, increases the reliability and security of routing information; Changes in the RIP routing updating by broadcast mechanism, more efficient instead of 255.255.255.255 broadcast address is used to send routing updates.RIPv2 downward compatible RIPv1.

\section{Algorithm implementation}

The establishment of the routing table: router when just beginning to work, only know to directly connected network distance (the distance is defined as 1). Later, each router also with only a very limited number of adjacent routers exchange routing information and update. After several times to update, all of the routers will know finally reached the autonomous system in any network shortest distance and the next-hop router's address. Concrete steps is given below:

Receive adjacent routers (its address X) as a RIP message:

Modify the RIP message of all items first: "next jump" in the address field are changed to X, and put all of the "distance+1" the value of the field.

Repeat the following steps to Modified RIP message every project

If the purpose of network of project is not in the routing table, then add the project to the routing table; Otherwise, if the next-hop router address field given is the same, have received the project to replace the original project in the routing table; Otherwise, if received project of distance is less than the distance in the routing table, the update; otherwise, do nothing.

\section{Application for example}

Application example: the known router B routing table are shown in table 1.

Table 1 the router B routing table

\begin{tabular}{ccc}
\hline Purpose of the network & distance & The next-hop router \\
\hline Net2 & 2 & $\mathrm{C}$ \\
Net6 & 8 & $\mathrm{~F}$ \\
Net8 & 4 & $\mathrm{E}$ \\
Net9 & 4 & $\mathrm{~F}$ \\
$\ldots$ & $\ldots$ & $\ldots$ \\
\hline
\end{tabular}

Now received from the neighboring router $C$ routing updates, as shown in table 2.

Try to find the updated router B routing table.

Answer: like a router, do not need to know the network topology. First the distance is added 1 in Table2, and the next hop router are changed to $\mathrm{C}$, have the following table3: 
Put the table 3 and table 1 each line of the comparison:

Net1 of Line1in table 3 not in table 1, so add this line to table 1;Net2 of Line2 in table 3 and in table1 and the next-hop address is C, distance increases, so will update;Net3 of Line3 in table 3 not in table 1 , add this line to table 1;Net6 of Line 4 of table 3 in table 1, but the next-hop router is different, to compare distance, the distance of new router is 2,less than 8 of the original table, therefore to update;Net8 of Line5 of table 3 in table 1, but the next-hop router is different, so to compare distance, the distance is 4 in new routers, originally in the table1 is 4, so there is no update;Net9 of Line6 of table 3 in table 1, but the next-hop router is different, so compare distance, distance is 6 in new routers, greater than in the original table in the distance, so there is no update.

Table 2 Routing updates from C

\begin{tabular}{ccc}
\hline Purpose of the network & distance & The next-hop router \\
\hline Net1 & 7 & $\mathrm{~A}$ \\
Net2 & 4 & $\mathrm{~F}$ \\
Net3 & 8 & E \\
Net6 & 1 & Direct delivery \\
Net8 & 3 & $\mathrm{~A}$ \\
Net9 & 5 & $\mathrm{~F}$ \\
$\ldots$ & $\ldots$ & $\ldots$ \\
\hline
\end{tabular}

Table 3 the modified Table 2

\begin{tabular}{ccc}
\hline Purpose of the network & distance & The next-hop router \\
\hline Net1 & 8 & $\mathrm{C}$ \\
Net2 & 5 & $\mathrm{C}$ \\
Net3 & 9 & $\mathrm{C}$ \\
Net6 & 2 & $\mathrm{C}$ \\
Net8 & 4 & $\mathrm{C}$ \\
Net9 & 6 & $\mathrm{C}$ \\
$\ldots$ & $\ldots$ & $\ldots$ \\
\hline
\end{tabular}

Therefore, it is concluded that the updated routing table as shown in table 4:

Table 4 the updated routing table B

\begin{tabular}{ccc}
\hline Purpose of the network & distance & The next-hop router \\
\hline Net1 & 8 & C \\
Net2 & 5 & C \\
Net3 & 9 & C \\
Net6 & 2 & C \\
Net8 & 4 & E \\
Net9 & 4 & F \\
$\ldots$ & $\ldots$ & $\ldots$ \\
\hline
\end{tabular}

\section{Summary}

Analyzes the advantages and disadvantages of RIP, RIP including RIPv1 and RIPv2 two versions. RIPv1 protocol standard is before VLSM and CIDR, so does not support VLSM and CIDR, in the routing updates, not including the subnet mask, in the VLSM and CIDR network interconnection environment RIPv1 cannot be started. RIPv2 RIPv1 is improved version, it retains the most characteristics of RIP, strengthen the function of support VLSM and CIDR, in the routing updates will send subnet mask information. 


\section{Acknowledgment}

Author's brief introduction: CHEN Jing (1981- ), woman, Qiongzhou University, Instructor, master, the research direction: Computer aided education, computer network. Tel: 18976000277.

This work was supported in part by a grant from the Scientific Research Foundation of Qiongzhou University (No. QYQN2013-26), the project of science foundation of educational department of Hainan province (613171).

\section{References}

[1] Lin Yuanguai compiled. The computer network experiment tutorial Beijing: mechanical industry press. June 2012.

[2] Xie Xiren. Computer network (version 6). Beijing: electronic industry press June 2013. 